\title{
Variación de la Adherencia de las Armaduras Polarizadas Católicamente en Morteros de Cemento con Escoria
}

\author{
Jorge L. Fernández, Jorge D. Sota, Ricardo O. Carbonari y Roberto Romagnoli \\ Universidad Nacional del Comahue, Facultad de Ingeniería, Departamento de Construcciones, \\ Buenos Aires 1400, (CP 8300) Neuquén-Argentina (e-mail: jlfernan@uncoma.edu.ar)
}

Recibido Ago. 05, 2009; Aceptado Sept. 08, 2009; Versión Final recibida Nov. 04, 2009

\begin{abstract}
Resumen
El objetivo de este trabajo es explorar el efecto de diferentes sobrepotenciales aplicados al acero (250 y $-500 \mathrm{mV}$ ) sobre la adherencia al mortero de cemento Portland adicionado con escoria granulada de alto horno (EGAH). Para realizar esta investigación se elaboraron probetas de mortero que fueron polarizadas católicamente, a los sobrepotenciales mencionados, durante 2 años en agua potable y cloruro de sodio $3 \%$. Una vez finalizado el período de ensayo se determinó la tensión de adherencia de las barras de acero por medio de un ensayo de arrancamiento. Los resultados obtenidos muestran que las variaciones de adherencia son, en general, aleatorias; observándose ligeros aumentos de la adherencia y en algunos casos una disminución que no superó el $5 \%$. La superficie de las barras de acero se observó por microscopía electrónica de barrido, comprobándose que la polarización modificó la película protectora.
\end{abstract}

Palabras clave: corrosión, armaduras, adherencia, escoria de alto horno, protección catódica

\section{Variation in Rebars Bond Strength in Cathodically Protected Mortar Specimens Containing Ground Blast Furnace Slag}

\begin{abstract}
The objective of this research was to investigate the effect of two applied overpotentials (-250 and $500 \mathrm{mV}$ ) on the adhesion of rebars in Portland cement mortars containing ground blast furnace slag. In order to carry out this investigation mortar slabs which were polarized, during 2 years, at the above mentioned overpotentials, in tap water and 3\% sodium chloride, were made. After this exposure period, rebars bond strength was determined by a pull out test. As a general rule, bond strength variations were found to be random, observing slight bond strength increasing and in some cases a deceasing that did not exceed 5\%. Rebars surface was also analyzed by scanning electron microscopy, finding that the protective layer on rebars changed as a consequence of the applied overpotentials.
\end{abstract}

Keywords: corrosion, rebars, adherence, ground blast furnace slag, cathodic protection 


\section{INTRODUCCION}

Los metales, en general, tienden a reaccionar químicamente con el medio circundante a fin de adquirir un estado de menor energía. En este sentido el hierro (o el acero) reacciona con el oxígeno del aire a fin de formar óxidos y disminuir, como se dijo, la energía del sistema. El hormigón constituye el medio ideal para preservar al acero de la oxidación natural pues la pasta cementicia provee las condiciones ideales para que se produzcan las reacciones necesarias que conducen a la formación de una película protectora sobre el metal. Esta película protectora es tal que preserva al acero mientras que las condiciones del medio ambiente no sean modificadas en forma acentuada, como, por ejemplo, el pH elevado de la pasta de cemento. Por otro lado, el recubrimiento de hormigón, cuando está bien diseñada en composición y espesor, funciona como una barrera mecánica que impide el ingreso de agentes agresivos del exterior tales como oxígeno, agua, cloruro, dióxido de carbono, etc. que pueden destruir la película protectora.

Cuando el hormigón no es de la calidad adecuada o no tiene el espesor suficiente, resulta necesario emplear ciertos procedimientos a fin de preservar a las armaduras de refuerzo de la corrosión. Entre éstos se encuentran los tratamientos superficiales del hormigón (pinturas, impregnaciones, etc.), las reparaciones, la protección catódica de las barras de refuerzo, etc. La protección catódica consiste en aplicar un potencial lo suficientemente negativo como para llevar al metal a la zona de inmunidad o a la de pasividad (Hausmann, 1969; Guillén, 1987; Marciana Mos et al., 2006).

La aplicación de la protección catódica produce modificaciones en la interfase acero-mortero que se traducen en cambios en la adherencia. Existe poca información bibliográfica sobre este tema y aún subsiste la polémica acerca de la pérdida de adherencia, especialmente cuando se llega al potencial necesario para desprender hidrógeno sobre la barra de acero. Para evitar este fenómeno Vrable y otros investigadores (Álvarez y Berardo, 1991; Vrable, 1980) sugieren que el potencial de polarización debe ser más positivo que $-1,1 \mathrm{~V}$ con respecto al ESC (electrodo de cobre/sulfato de cobre). Tache y Lemoine (Guillén, 1987; Tache y Lemoine,1992) sostienen que la polarización de las armaduras no afecta la adherencia del acero al mortero durante un período de ensayo de 300 días y potenciales de protección comprendidos entre -900 y $-1500 \mathrm{mV}$ medidos con respecto al ECS (electrodo de calomel saturado). El valor de la adherencia resultó independiente del tipo de superficie expuesta, ya sea con óxidos de laminación, arenada o en presencia de corrosión inducida por una fuerte polarización anódica. Estos resultados no coinciden con otras investigaciones realizadas que sugieren pérdidas elevadas para sobrepotenciales altos (Rasheeduzzafar et al., 2007)

Existen varios criterios para la selección del potencial adecuado de protección (Álvarez y Berardo, 1991; Vrable y Wilde, 1980; Gummow, 1986; Rasheeduzzafar, 1992; Mudd et al., 1988; Sosa et al., 2007). Uno de los más difundidos es el que propone un potencial de polarización de $-850 \mathrm{mV}$ respecto del ESC (Vrable y Wilde, 1980; Tache y Lemoine, 1992; Gummow, 1986); una alternativa de este criterio es tomar el valor de $-850 \mathrm{mV}$ al corte de la polarización, a fin de descontar el sobrepotencial óhmico (Rasheeduzzafar, 1992). Los otros criterios toman como punto de partida el potencial de corrosión de la armadura y consideran que el de protección debería estar desplazado 100, $-300 \mathrm{mV}$, etc. Sin embargo, el potencial a circuito abierto de las armaduras puede variar de un punto a otro de la estructura y con las condiciones ambientales (Gowers y Millard, 1993; Wami et al., 1993), de manera que no constituye un punto de referencia válido. De acuerdo a estudios previos (Vetere et al., 1996), para realizar esta investigación se seleccionaron dos sobrepotenciales de polarización para el acero en mortero, -250 y $-500 \mathrm{mV}$, tomados con respecto al potencial de corrosión del acero en agua potable $(-725 \mathrm{mV})$ y en cloruro de sodio al $3 \%$ (-750 mV vs. ECS), respectivamente. El sobrepotencial de $-500 \mathrm{mV}$ es suficiente para desprender hidrógeno.

Para efectuar la medida de adherencia acero-mortero se desarrolló, a partir de normas conocidas, un ensayo de arrancamiento que pudiera aplicarse a este caso particular y que realizado en forma comparativa con muestras no polarizadas mostrara la variación relativa de la adherencia de las barras de acero en probetas de mortero. Los resultados obtenidos muestran que para el sobrepotencial más alto puede haber pérdida de adherencia, no superior al $10 \%$. La presencia de EGAH resultó ser beneficiosa para reducir el proceso de corrosión tanto en las probetas testigo como en las que fueron polarizadas catódicamente. El objetivo de este trabajo fue estudiar la variación de 
la adherencia en la interfase acero-mortero de probetas polarizadas catódicamente con dos sobrepotenciales (uno que corresponde a la zona de desprendimiento de hidrógeno), en dos electrolitos (agua potable y cloruro de sodio 3\%) y durante 2 años. El cemento utilizado para elaborar las probetas contenía escoria granulada de alto horno (EGAH).

\section{MATERIALES Y METODOS}

Las probetas de acero-mortero para este estudio eran cilíndricas de $5 \times 10 \mathrm{~cm}$. Se prepararon utilizando cemento portland normal adicionado con EGAH y arena natural (Norma IRAM 1633) en una relación 1:3 en peso y dos razones agua cemento (a/c) 0,50 y 0,65, en peso, respectivamente. Se curaron durante 28 días bajo agua con cal, de acuerdo a un procedimiento descrito en un trabajo previo (Vetere et al., 1998). Los ensayos se hicieron en forma comparativa con probetas elaboradas con cemento portland normal.

Conjuntos de ocho probetas fueron polarizados, cada uno, a los sobrepotenciales mencionados, para ser sometidos, luego, al ensayo de arrancamiento a fin determinar la variación de adherencia de la interfase acero-mortero luego de dos años de exposición. Las probetas se colocaron en cubas de material plástico de $60 \times 40 \times 25 \mathrm{~cm}$ con ánodos de platino; se eligieron dos electrolitos, agua potable, de resistividad moderadamente alta, y cloruro de sodio $3 \%$, agresivo al acero de las armaduras y de baja resistividad. Conjuntos similares de probetas permanecieron sin polarizar, en los mismos electrolitos, a fin de ser utilizados como ensayo en blanco. El circuito de polarización empleado fue descrito en un trabajo previo (Vetere et al., 1998). Por otro lado, a fin de estimar el grado de protección logrado con los sobrepotenciales aplicados, se midió la disminución de la velocidad de corrosión para varios sobrepotenciales de polarización, colocando paneles de acero de similar composición, de $20 \mathrm{~cm}^{2}$ de superficie, en la solución más agresiva, cloruro de sodio $3 \%$ (termostatizada a $2{ }^{\circ} \mathrm{C}$ ), durante 5 días. Se determinó la cantidad de hierro que reaccionó por absorción atómica, previa disolución de los productos de corrosión en $\mathrm{HCl} 10 \%$, protegiendo catódicamente la barra de acero con una densidad de corriente $50 \mathrm{~mA} . \mathrm{cm}^{-2}$.

El ensayo de adherencia, utilizado también por otros investigadores (Ihekwalva et al., 1996), consiste en extraer una barra de acero empotrada en el mortero, por la aplicación de un esfuerzo creciente a fin de producir el arrancamiento ("pull out") de la barra de la probeta de mortero. Este ensayo se desarrolló utilizando como base las normas ASTM C 234-91a e IRAM 1596/56, siendo las principales modificaciones la reducción del tamaño de la probeta $(5 \times 10 \mathrm{~cm})$ y el diámetro de la barra de acero $(6 \mathrm{~mm})$. La rama larga de la barra empotrada fue tomada por la mordaza de la máquina de ensayo; la pequeña saliente de longitud $4-5 \mathrm{~mm}$, del otro extremo, sirvió para registrar los deslizamientos del acero con respecto al mortero por medio de un micrómetro (sensibilidad de medida 0,01 mm.). Entre la superficie de la probeta de mortero y la base rígida de la máquina de ensayo se colocó un material de nivelación y transferencia para evitar concentración de tensiones localizadas. El ensayo se realizó aplicando una carga $P$, aproximadamente 20 a $30 \mathrm{Kg} \cdot \mathrm{cm}^{-2} \cdot \mathrm{min}^{-1}$; registrándose las cargas necesarias para producir deslizamientos de 0,1;0,2;0,3;0,4;0,5 mm (o más en caso de ser posible) y determinar la carga máxima de arrancamiento. Se pueden presentar tres tipos de rotura que indican la finalización del ensayo: que la barra deslice por lo menos $2,5 \mathrm{~mm}$ respecto al mortero, que se rompa el mortero de la probeta por resultar la tensión de adherencia superior a la resistencia a tracción del mortero o que la barra de acero llegue a fluencia o se rompa; en este estudio se trató que la rotura se produzca en la forma descripta en primer término.

La tensión de adherencia, $\mathrm{T}_{\text {adh }}\left(\mathrm{Kg} \cdot \mathrm{cm}^{-2}\right)$, se calcula utilizando la expresión:

$T_{a d h}=\frac{P_{(\mathrm{kg})}}{S_{\left(\mathrm{cm}^{2}\right)}}$

siendo $\mathrm{P}$ la carga necesaria para producir un determinado deslizamiento y $\mathrm{S}$ la superficie lateral de la barra de acero incluida en el mortero. 
En la presente investigación se determinó la $T_{\text {adh }}$ como un promedio de las cargas necesarias para producir las deformaciones en intervalos de 0,10 a 0,5 mm. Se ensayaron 4 probetas por edad, por cada electrolito y por cada sobrepotencial de polarización. Por tratarse de ensayos comparativos, se considera que los módulos de elasticidad individuales del mortero y del acero se mantienen constantes para todas las probetas. Las varillas de acero incluidas en las probetas de mortero, polarizadas durante 2 años en las condiciones especificadas, fueron inspeccionadas visualmente a fin de determinar el grado de corrosión de las armaduras, luego de efectuarse el ensayo de adherencia. También se observó la superficie de las mismas en el microscopio electrónico

\section{RESULTADOS Y DISCUSION}

\section{Polarización de las probetas}

El potencial de las probetas de acero en el mortero fue monitoreado en forma continua durante los dos años de exposición. Para el valor más alto de sobrepotencial, debido a las características voltamperométricas del sistema, se logró una rápida estabilización del potencial. Los datos experimentales de la Tabla 1 se ajustan con la ecuación empírica:

$M=M_{0} e^{0,036 s}$

cuya forma matemática es similar a la de las expresiones de Tafel para el cálculo de la velocidad de corrosión (Shreir, 1976), donde M es la velocidad de corrosión expresada en mgFe.cm ${ }^{-2}$.año-1 para el sobrepotencial aplicado (S) y $\mathrm{M}_{0}$ es la velocidad de corrosión, expresada en las mismas unidades, en ausencia de campo eléctrico. De acuerdo a los mencionados valores, se puede decir que a partir de un sobrepotencial de $-250 \mathrm{mV}$, la velocidad de corrosión del acero es despreciable.

Tabla 1: Disminución de la velocidad de corrosión del acero en función del sobrepotencial aplicado

\begin{tabular}{|c|c|c|}
\hline $\begin{array}{c}\text { Sobrepotencial } \\
\text { aplicado }(\mathrm{mV})\end{array}$ & mg de Fe disueltos & $\begin{array}{c}\text { Reducción relativa de la } \\
\text { velocidad de corrosión }\end{array}$ \\
\hline 0 & 107 & --- \\
\hline-50 & 16,0 & 85,0 \\
\hline-100 & 2,65 & 97,5 \\
\hline-150 & 0,48 & 99,6 \\
\hline-200 & 0,08 & 99,9 \\
\hline-250 & 0,01 & $\cong 100$ \\
\hline
\end{tabular}

\section{Ensayo de adherencia acero-mortero}

La polarización de las barras no afectó significativamente la adherencia de las mismas al mortero y las variaciones observadas son más bien aleatorias. De acuerdo a los valores que se consignan en las Tablas 2 y 3, en general, la resistencia mecánica de adherencia aumenta ligeramente, 3-7\%, con respecto a los patrones, para ambos sobrepotenciales. En algunos casos se observó una disminución de la adherencia, particularmente para la razón a/c 0,65, la cual no superó, en el peor de los casos, el $5 \%$.

En la tabla 2 se consigna la variación porcentual de la adherencia con respecto a los patrones que son las probetas de acero mortero sin polarizar sumergidas en los dos electrolitos propuestos. En la tabla 3 se consigna la variación porcentual de la adherencia con respecto a los patrones que son las probetas de acero mortero sin polarizar sumergidas en los dos electrolitos propuestos. 
Tabla 2: Ensayo de adherencia acero-mortero de cemento portland con EGAH. Razón agua / cemento 0,50

\begin{tabular}{|c|c|c|c|c|}
\hline Tipo de mortero & $\begin{array}{c}\text { Sobrepotencia } \\
\text { I aplicado }\end{array}$ & Electrolito & $\begin{array}{c}\text { Tensión de adherencia } \\
\text { Kg.cm }\end{array}$ & $\begin{array}{c}\% \\
\text { variación }\end{array}$ \\
\hline \multirow{3}{*}{ Sin escoria } & 0 & Agua & 89,0 & --- \\
& -250 & potable & 91,5 & +3 \\
& -500 & & 95,5 & +7 \\
\hline \multirow{3}{*}{ Sin escoria } & 0 & NaCl 3\% & 80,0 & --- \\
& -250 & & 80,0 & 0 \\
& -500 & Agua & 82,5 & +3 \\
\hline \multirow{3}{*}{ Con escoria } & 0 & potable & 63,5 & +- \\
& -250 & & 66,0 & +4 \\
& -500 & NaCl 3\% & 68,0 & +7 \\
\hline \multirow{2}{*}{ Con escoria } & 0 & & 58,0 & +- \\
& -250 & 60,0 & +3 \\
\hline
\end{tabular}

Tabla 3: Ensayo de adherencia acero-mortero de cemento portland con EGAH. Razón agua / cemento 0,65

\begin{tabular}{|c|c|c|c|c|}
\hline Tipo de mortero & $\begin{array}{l}\text { Sobrepotencial } \\
\text { aplicado }\end{array}$ & Electrolito & $\begin{array}{l}\text { Tensión de adherencia } \\
\qquad \mathrm{Kg} \cdot \mathrm{cm}^{-2}\end{array}$ & \% variación \\
\hline \multirow{3}{*}{ Sin escoria } & 0 & \multirow{3}{*}{$\begin{array}{c}\text { Agua } \\
\text { potable }\end{array}$} & 41,0 & --- \\
\hline & 250 & & 40,0 & $-2,5$ \\
\hline & 500 & & 43,5 & $+6,0$ \\
\hline \multirow{3}{*}{ Sin escoria } & 0 & \multirow{3}{*}{$\mathrm{NaCl} 3 \%$} & 34,5 & --- \\
\hline & 250 & & 35,0 & +1 \\
\hline & 500 & & 32,8 & -5 \\
\hline \multirow{3}{*}{ Con escoria } & 0 & \multirow{3}{*}{$\begin{array}{c}\text { Agua } \\
\text { potable }\end{array}$} & 48,0 & --- \\
\hline & 250 & & 47,0 & -2 \\
\hline & 500 & & 48,5 & +1 \\
\hline \multirow{3}{*}{ Con escoria } & 0 & \multirow{3}{*}{$\mathrm{NaCl} 3 \%$} & 36,0 & --- \\
\hline & 250 & & 35,5 & -1 \\
\hline & 500 & & 38,5 & +7 \\
\hline
\end{tabular}

Si bien el sobrepotencial de $-250 \mathrm{mV}$ fue suficiente para detener el proceso de corrosión del acero desnudo (Tabla 1), sin la cubierta cementicea, no resultó suficiente para evitar la corrosión de las armaduras en el mortero, particularmente de las que estaban en la solución de $\mathrm{NaCl} 3 \%$ ". Cuando las barras fueron polarizadas y extraídas de las probetas se observó corrosión localizada, (Ogura y Takesue, 1980; Sekar et al., 2007). A sobrepotenciales de $-500 \mathrm{mV}$ el proceso de corrosión fue sensiblemente menor y menor aún en presencia de EGAH. De esta manera, se concluye que para empezar a lograr una protección eficiente se necesitan, al menos, sobrepotenciales de $-500 \mathrm{mV}$ aunque existe el riesgo de tener pérdidas de adherencia del orden de 5\%. 


\section{Análisis de la superficie de las barras de acero por microscopía electrónica}

La película protectora que estaba sobre las barras de acero tenía rasgos similares en todos los casos (Romagnoli et al., 1998), en cuanto al tipo de compuestos que se detectaron, independientemente de la adición de EGAH. La muestras con mayor razón a/c presentaron mayor cantidad de formaciones de óxido de hierro, especialmente las estacionadas en $\mathrm{NaCl}$ 3\%. La presencia de EGAH disminuyó sensiblemente la formación de óxidos de hierro.

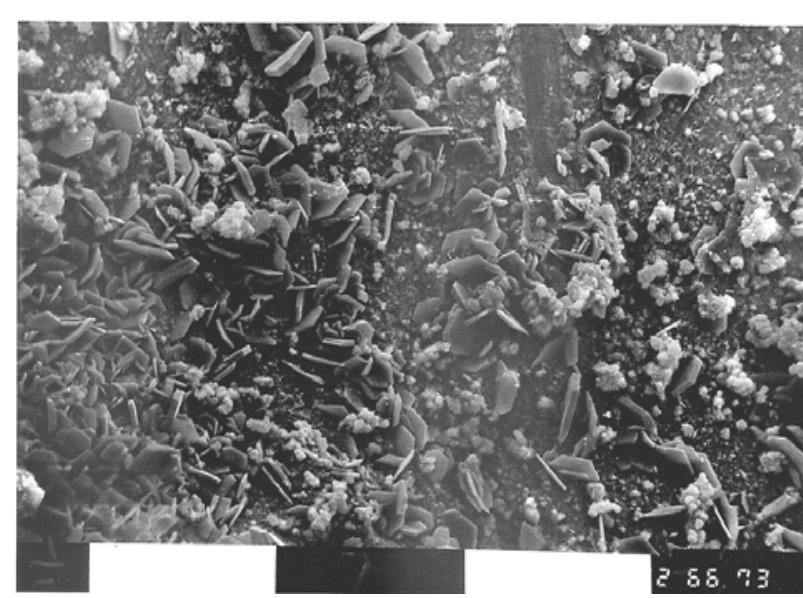

Fig. 1: Micrografía de la película protectora de las barras de acero en morteros con EGAH (300X) en agua potable.

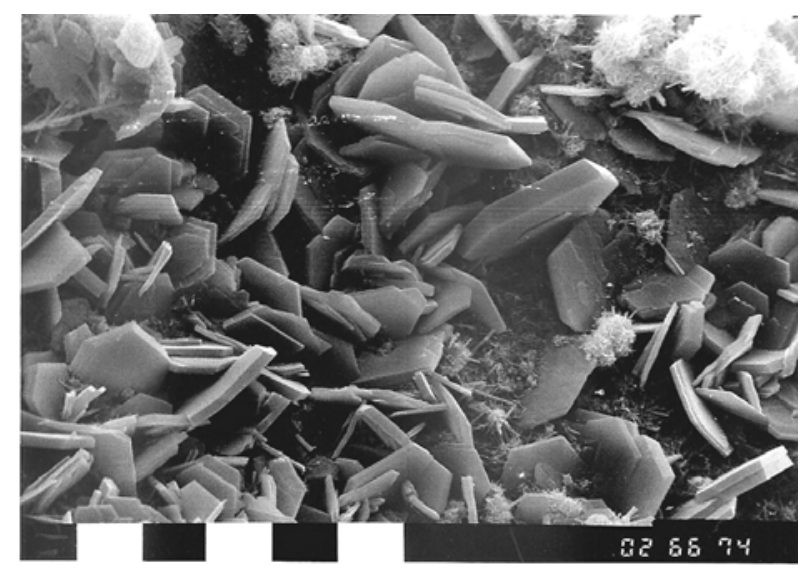

Fig. 3: Micrografía de las placas con alto contenido de alúmina sobre la película protectora de las barras de acero en morteros con EGAH (1000X).

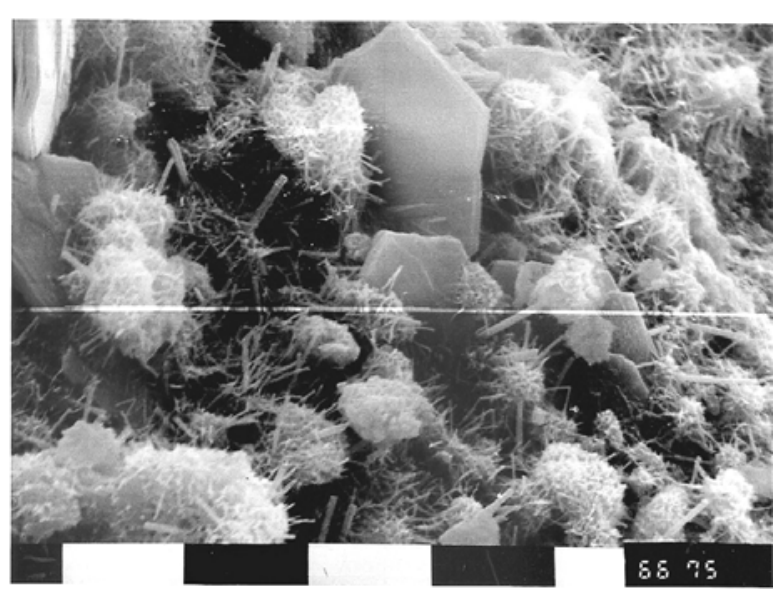

Fig. 2: Micrografía de la película pulverulenta sobre las barras de acero en morteros con EGAH (2000X) en agua potable.

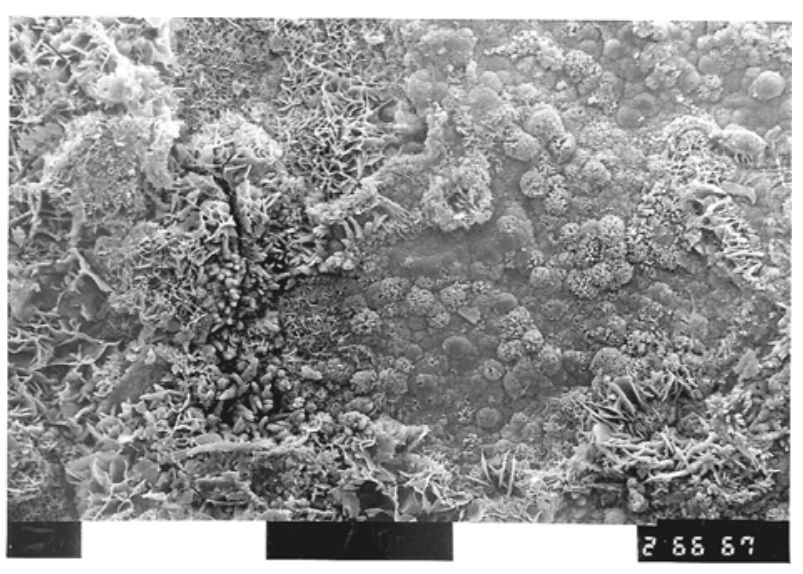

Fig. 4: Micrografía de los óxidos de hierro crecidos sobre la película protectora del acero polarizado, en morteros sin EGAH (300X), en $\mathrm{NaCl} 3 \%$.

A manera de ejemplo, se describe la morfología y composición de película protectora sobre las barras con razón a/c 0,65, sin polarizar y polarizadas con el sobrepotencial más bajo empleado. La película protectora de las barras que no fueron polarizadas, almacenadas en agua potable, estaba constituida por abundante silicato de calcio hidratado $(\mathrm{SCH})$ amorfo junto con algo de óxido de hierro y formaciones pulverulentas (Fig. 1). El contenido promedio de los componentes mayoritarios sobre la película protectora era: $\mathrm{Al}_{2} \mathrm{O}_{3}: 11,29 \%$; $\mathrm{SiO}_{2}: 18,87 \%$; $\mathrm{CaO}: 28,25 \%$ y $\mathrm{Fe}_{2} \mathrm{O}_{3}: 41,49 \%$. La presencia de óxido de hierro se atribuye a la porosidad algo elevada de los morteros con razón a/c 0,65. Las formaciones pulverulentas (Fig. 2) son más ricas en $\mathrm{SiO}_{2}$ y $\mathrm{CaO}$, siendo la composición promedio: $\mathrm{Al}_{2} \mathrm{O}_{3}$ : 8,68\%; $\mathrm{SiO}_{2}$ : 36,29\%; $\mathrm{CaO}: 32,26 \%$ y $\mathrm{Fe}_{2} \mathrm{O}_{3}: 22,77 \%$. En el caso de los morteros con EGAH se observaron placas con alto contenido de aluminio: $\mathrm{Al}_{2} \mathrm{O}_{3}: 21,80 \%$; $\mathrm{SiO}_{2}$ : 11,80\%; $\mathrm{CaO}: 39,98 \%$ y $\mathrm{Fe}_{2} \mathrm{O}_{3}: 26,41 \%$ (Fig. 3). En las barras que estuvieron en contacto con la solución de $\mathrm{NaCl} 3 \%$, en 
probetas sin EGAH, se observó un aumento importante en el contenido de óxidos de hierro $(88,29 \%)$ sobre la película pasiva (Fig. 4).

La polarización de las barras introduce algunos cambios en la película protectora A pesar del sobrepotencial aplicado todavía se observó la presencia de óxido de hierro aunque en menor proporción que en las barras no polarizadas. En las micrografías de las barras polarizadas, sin EGAH, aparecieron algunas formaciones diferenciales como las de la Fig. 5, las cuales pueden apreciarse con más detalle en la Fig. 6. En presencia de EGAH no se observaron las placas ricas en aluminio.

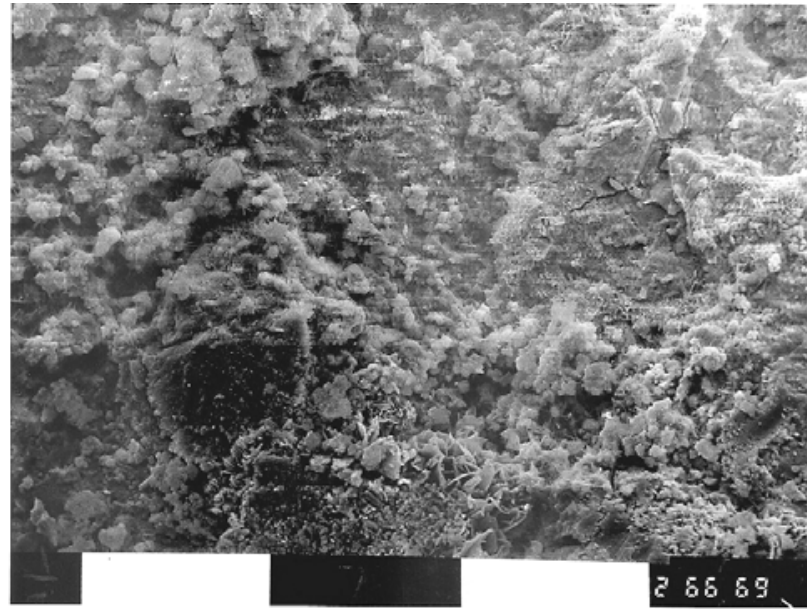

Fig. 5: Micrografía de la película protectora sobre las barras de acero polarizadas (-250 $\mathrm{mV}$ ) en morteros con EGAH (300X), en agua potable.

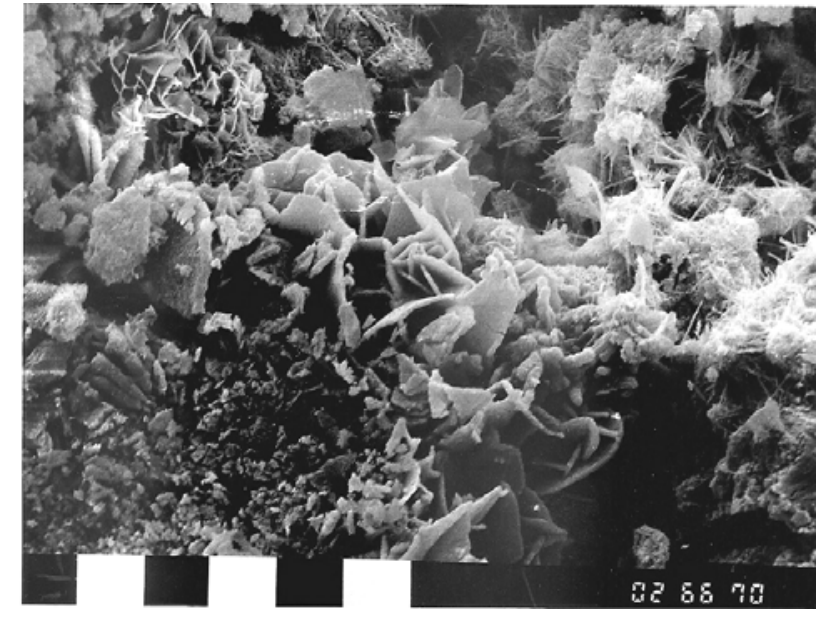

Fig. 6: Micrografía de la película protectora sobre las barras de acero polarizadas (-250 $\mathrm{mV}$ ) en morteros con EGAH (1000X), en agua potable.

\section{CONCLUSIONES}

De acuerdo a las experiencias realizadas, al cabo de dos años, se puede concluir que el sobrepotencial de $-250 \mathrm{mV}$ resultó insuficiente para proteger a las barras de acero en las probetas de mortero estacionadas en cloruro de sodio 3\%, observándose una corrosión de las armaduras expuestas. La variación de la adherencia acero-mortero observada al cabo de dos años no implica una pérdida significativa. En el peor de los casos es del orden del $5 \%$ con respecto a los patrones no polarizados. La presencia de EGAH en el mortero fue beneficiosa en el sentido de que la corrosión observada en las barras de acero fue menor.

\section{AGRADECIMIENTOS}

A las siguientes instituciones para realizar esta investigación: UNLP (Universidad Nacional de la Plata), UNCOMA (Universidad Nacional del Comahue), CONICET (Consejo nacional de Investigaciones Científicas y Técnicas) y CIC (Comisión de Investigaciones Científicas de la Provincia de Buenos Aires).

\section{REFERENCIAS}

Álvarez, N. y Berardo, L., Protección catódica en estructuras de hormigón armado, Monografía INTI, Setiembre, p.p. 11-22, Buenos Aires, Argentina (1991).

Gowers, K.R. y Millard, S.G., On-site linear polarization resistance mapping of reinforced concrete structures, Corr. Sci. 35 (5-8) 1593-1600 (1993).

Guillén, M.A., Nuevos campos de aplicación de la protección catódica, Rev. Iber. Corros. y Prot. XVIII (2-6) 99-105 (1987). 
Gummow, R.A., Cathodic protection criteria. A critical review of NACE Standard RP-01-69, Mat. Performance 25(9) 9-16 (1986).

Hausmann, D.A., Criteria for cathodic protection of steel in concrete, Mat. Protection 8(10)23-25 (1969).

Ihekwalva, N.M., Hope, B.B. y Hansson, C.M., Pull-out and bond degradation of steel rebars in ECE Concrete, Cement and Concrete Research, 26(2) 267-282 (1996).

IRAM 1633, Instituto Argentino de Racionalización de Materiales, Arena Normal, 3-13, Buenos Aires, Argentina (1965).

Marciana Mos, D., González Ramírez, P.M., Vaswani Reboso, J., Santana Hernández, F.J., Betancor Alemán, J., Santana Rodríguez, J.J., González, J.E., Armaduras de hormigón y técnicas de protección catódica, Perspectiva de la investigación sobre materiales en España en el siglo XXI, Vol. 2, p.p. 737-740 (2006).

Mudd, C.J., Mussinelli, G.L., Tettamanti, M. y Pedeferri, P., Cathodic Protection of steel in concrete, Mat. Performance, 27(9) 18-24 (1988).

Ogura, K. y Takesue, N., Pit formation in the cathodic polarization of passive iron I. Dissolution of passive film and pit initiation, Corrosion (NACE), 36(9) 487-493 (1980) .

Rasheeduzzafar, Ali M.G., Polarization period, current density and the cathodic protection criteria, ACl Materials Journal, May-June, Title Nº 89-M27 247-251 (1992).

Rasheeduzzafar, Ali, M.G.; Alsulaimani, G.J., Degradation of bond between reinforcing steel and concrete due to cathodic protection current, ACI Materials Journal; 90, 8-15 (2007)

Romagnoli, R., Vetere, V.F., Sota, J.D., Maiza, P.J., Marfil, S., Lucchini, I.T., Batic, O.R. y Carbonari, R.O., Análisis de la interfase acero-mortero en probetas polarizadas catódicamente, Proceedings del $1^{\text {st }}$ International Congress of Concrete Technology, 2-4 de junio, Buenos Aires, Argentina (1998).

Sekar, A.S.S., Saraswathy, V., Parthiban, G.T., Cathodic protection of steel in concrete using conductive polymer overlays, Int. J. Electrochem. Sci., 2, 872-882 (2007).

Shreir, L.L., Outline of Electrochemistry, Corrosion, vol. 1, The Butterworth Group, 9:1-9:124, England (1976).

Sosa, M., Camacho, R., Perez, T., Gonzalez-Sanchez, J., Cathodic protection of concrete structures containing calcareous aggregates in tropical-humid marine environments, Anti-Corrosion Methods and Materials, 54(2), 103-110 (2007).

Tache, G. y Lemoine, L., Protection cathodique des armatures du beton armé en mer: étude experimental des criteres et des effects, Pub. CNEXO (Act. Colloq.) N 15, Paris, Francia (1992).

Vetere, V.F., Batic, O.R., Romagnoli, R., Lucchini, I.T., Sota, J.D. y Carbonari, R.O., Evaluación electroquímica de los criterios de protección catódica del acero en el hormigón, Anales de las Jornadas SAM'96 245-248, Argentina (1996).

Vetere, V.F., Romagnoli, R., Sota, J.D., Lucchini, I.T., Carbonari, R.O. y Batic, O.R., Modificación del circuito de corriente impresa para protección catódica para un mejor control del potencial en la interfase acero-mortero, Proceedings del $1^{\text {st }}$ International Congress of Concrete Technology, 2-4 de junio, Buenos Aires, Argentina (1998).

Vrable, J.B. y Wilde, B.E., Electrical requirements for cathodic protection of steel in simulated concrete environments, Corrosion (NACE), 36(1) 18-23 (1980).

Wami, H., Kasai, H., Uchibori, T. y Kataoka, K., New corrosion diagnosis system and its applicability to existing structures, Durability of building materials and components pp. 893-902 (1993). 